[postprint version]

\title{
Women's help-seeking behaviors for depressive symptoms during the perinatal period: Socio- demographic and clinical correlates and perceived barriers to seeking professional help
}

\author{
Ana Fonseca, Ricardo Gorayeb, \& Maria Cristina Canavarro
}

\begin{abstract}
Objective: This study aims to characterize the help-seeking behaviors of women who were screened positive for perinatal depression, to investigate its sociodemographic and clinical correlates, and to characterize the perceived barriers that prevent women from seeking professional help.

Design: Cross-sectional internet survey.

Setting: Participants were recruited through advertisements published in pamphlets and posted on social media websites (e.g., Facebook) and websites and forums that focused on pregnancy and childbirth.

Participants: 656 women (currently pregnant or who had a baby during the last 12 months) completed the survey.

Measurements: Participants were assessed with the Edinburgh Postpartum Depression Scale, and were questioned about sociodemographic and clinical data, help-seeking behaviors and perceived barriers to help-seeking.

Findings: Different pathways of help-seeking were found, with only $13.6 \%$ of women with a perinatal depression seeking help for their emotional problems. Married women, currently pregnant women, and women without history of psychological problems had a higher likelihood of not engaging in any type of help-seeking behavior. The majority of women who had not sought professional assistance identified several barriers to help-seeking, particularly knowledge barriers.
\end{abstract}

Key Conclusions: Strategies to increase women's help-seeking behaviors should be implemented, namely improving mental health literacy, introducing screening procedures for mental health problems in pre/postnatal healthcare settings, and offering women innovative opportunities (e.g., web-based tools) that allow them to overcome the practical barriers to help-seeking.

Keywords: Barriers to care; Help-Seeking behaviors; Perinatal depression; Perinatal period; Survey. 


\section{Introduction}

Perinatal depression is generally recognized as a prevalent clinical condition, with incidence estimates of depressive symptoms among women reaching $14.5 \%$ during pregnancy and $49 \%$ during the first postpartum year (Gaynes et al. 2005). The estimated prevalence rate of postpartum depression in several countries, including Portugal, is 13\% (Gorman et al., 2004; O'Hara and Swain, 1996). However, this estimate may not be representative of the actual global prevalence and magnitude of the problem, as studies including non-western countries report prevalence estimates of postpartum depression from 0 to almost 60\%, suggesting cross-cultural and social diversity (Hakbreich and Karkun, 2006). Two reasons justify the need to intervene early and effectively in perinatal depression. First, its well-documented adverse effects: poor maternal health (Muzik and Borovska 2010), impairments in fetal development, neonatal outcomes (e.g., low birthweight; Accortt et al. 2015; Field et al. 2006) and infant development (Kingston et al. 2012; Kingston et al. 2015; Van den Bergh et al. 2005), disturbances in mother-child interactions (Field 2010; Righetti-Veltema et al. 2002; Tronick and Reck 2009), and higher levels of paternal depression (Goodman 2008). Second, the persistent nature of depressive symptomatology during the perinatal period. Not only is depression during pregnancy a risk factor for postpartum depression (Robertsonet al. 2004), but also women who develop depressive symptoms during the first months postbirth were found to have an increased risk of persistent depression throughout the first postpartum year (Beeghly et al. 2002).

Despite the negative consequences and pervasive nature of perinatal depression, research shows that few women (20\%-40\%) proactively seek professional assistance concerning their perinatal depression symptoms, although treatment is available (Dennis and Chung-Lee 2006; Henshaw 2004; McGarry et al. 2009; McIntosh 1993; Patel and Wisner 2011). In Portugal, no screening procedures are implemented to improve case identification and early intervention for perinatal depression. Women are routinely followed in obstetric appointments. Mental healthcare is provided only when women seek it, or when health professionals identify symptoms worthy of clinical attention, making access to intervention dependent on women's help-seeking behaviors and their ability to communicate with health professionals about their perinatal depressive symptoms (Brown and Limley, 2000). Understanding women's help- 
seeking behaviors is critical to devising strategies that increase treatment rates for perinatal depression; however, several questions related to this topic remain understudied.

First, it is important to understand these women's pathways of help-seeking behaviors. When exploring women's help-seeking behaviors, most studies focused only on one type of behavior: seeking professional assistance (e.g., seeking treatment from a health or mental health professional). However, the literature has highlighted that health care-seeking involves a series of stages or phases, beginning with the individual's recognition/awareness of the emotional problem, followed by the involvement of the individual's immediate social environment (e.g., discussing the problem with relatives and friends, sharing symptoms, and expressing need for support) and his/her willingness to seek professional assistance, and ending with an effective search for professional assistance so that the individual can address his/her emotional problems and make an informed decision about the available treatment options (Cauce et al. 2002; Kadushin 1958; Rickwood et al. 2005). As stated by Rickwood et al. (2005), the helpseeking process is a process "whereby the personal becomes increasingly interpersonal" (p. 8).

To our knowledge, only two studies on perinatal depression explored the diversity of women's help-seeking behaviors. Whitton et al. (1996) found that although 97\% of clinically depressed women recognized that they had been feeling worse than usual during the postnatal period, only $32 \%$ of them believed that they were suffering from postpartum depression. Fifty-four percent of these women spoke to their social network about their emotional problems, and $60 \%$ considered the possibility of seeking psychological treatment. Similarly, a recent study found that $88.9 \%$ of women with clinical levels of anxiety or depression considered that they had an emotional problem, $83 \%$ discussed it with their social network and $69 \%$ considered the option of seeking professional assistance, although only $33 \%$ of these women attended an appointment with a mental health professional (Henshaw et al. 2013). It is important not only to assess the proportions of women's different help-seeking behaviors during the perinatal period, but also to investigate the different pathways of help-seeking behaviors concerning women's perinatal depressive symptoms.

Second, it is important to examine the socio-demographic and clinical profiles of women who engage and women who do not engage in help-seeking behaviors concerning their perinatal depressive symptoms, because this may allow for the early identification of target populations (i.e., women with a higher likelihood of not seeking help if facing emotional problems during the perinatal period) for specific interventions to increase women's help-seeking behaviors and use of available treatment services 
for perinatal depression (McGarry et al. 2009). The few existing studies suggest that younger and less educated women from urban areas are more likely not to seek professional help (Herrick 2002; McGarry et al. 2009). Although no differences in other help-seeking behaviors were found regarding social class, women of higher social classes are less likely to recognize their symptoms (Whitton et al. 1996). Primiparity also seems to be associated with women's poor recognition of their symptoms (Whitton et al. 1996) and a lower likelihood of seeking professional help (Herrick 2002). McGarry et al. (2009) also found that depressed mothers who have had their infant admitted to the neonatal intensive care unit and who have sought help for depression during pregnancy are more likely to seek professional help for their perinatal depressive symptoms after childbirth. However, the role of other important clinical variables (e.g., psychiatric history) has not been explored yet, and the existing studies do not clearly allow for the identification of the socio-demographic and clinical characteristics of women who engage and those who do not engage in different help-seeking behaviors.

Moreover, a set of studies has identified several barriers that women perceive as preventing them from seeking professional help for their emotional problems, namely knowledge, attitudinal and practical/structural barriers (O'Mahen and Flynn 2008). Concerning knowledge barriers, women report having a poor recognition of depressive symptoms (Buist et al. 2005), including difficulty in distinguishing between the emotional maladjustment associated with the transition to parenthood and depression (Bilszta et al. 2010) and difficulty in assessing the severity of depressive symptoms (McCarthy and McMahon 2008). This poor recognition of symptoms may lead to difficulties in identifying help needs (Goodman and Tyer-Viola 2010). Moreover, women also report having scarce knowledge of the available services, treatment options and their benefits (Bilszta et al. 2010; Byatt et al. 2012; Henshaw et al. 2013).

Concerning attitudinal barriers, the feelings of guilt, shame and stigma that are associated with perinatal depression may prevent women from seeking professional help. Specifically, they fear being labeled mentally ill and/or bad mothers and experiencing their social network's disapproval (Bilszta et al. 2010; Callister et al. 2011; McIntosh 1993), and they may also feel embarrassed and uncomfortable when sharing their difficulties with health professionals (Callister et al. 2011; Woolhouse et al. 2009). Finally, a few practical/structural barriers were also identified in the literature, namely women's financial difficulties and work constraints, childcare responsibilities and limited access to healthcare (Callister et al. 2011; Dennis and Chung-Lee 2006; O'Mahen and Flynn 2008). However, with the exception of the study 
by O'Mahen and Flynn (2008), which found that women report more concern with structural/practical barriers than with knowledge and attitudinal barriers, there have been no other studies investigating the relative importance of the different barriers in preventing women from seeking professional help for their emotional/psychological problems during the perinatal period. Moreover, to our knowledge, there have been no studies examining the socio-demographic and clinical characteristics of women who perceive more barriers to them seeking professional assistance for their perinatal depressive symptoms; this knowledge would allow the early identification of women who may require particular attention from health professionals so that efforts can be developed for women to overcome these barriers.

This study focused on women who had a positive screening for perinatal depression and aimed: 1) to characterize women's pathways of help-seeking behaviors; 2) to investigate the sociodemographic and clinical correlates of the different help-seeking behaviors; 3) to assess the number and the relative importance of the perceived barriers that prevent women from seeking professional assistance (among women who had not sought professional assistance); and 4) to investigate the sociodemographic and clinical correlates of the different perceived barriers that prevent women from seeking professional assistance.

\section{Methods}

Procedure

This study was based on a cross-sectional internet survey that was conducted in Portugal. One of its primary goals was to examine women's help-seeking behaviors regarding perinatal depressive symptoms during the perinatal period. This study followed the ethical standards and procedures for research with human beings (e.g., Helsinki Declaration, World Medical Association 2000; American Psychological Association 2010) and was approved by the Ethics Committee of X. Participants were a self-selected online sample who replied to advertisements published in pamphlets that were distributed in several places in the central region of the country (e.g., maternity, pharmacies, etc.), and posted on social media websites (e.g., Facebook) and websites and forums focusing on pregnancy and childbirth (e.g., Rede Mãe, Forum PinkBlue). Advertisements about the study contained a weblink to the online survey (hosted by http://www.limesurvey.com/). Participation was voluntary and no remuneration was provided.

Women were eligible to participate in the study if they were: 1) currently pregnant or had given birth during the last 12 months, and 2) 18 years or older. At the beginning of the survey, women were 
informed of the goals of the study, the guarantee of anonymity and confidentiality, the voluntary nature of their participation and the possibility of dropping out of the study at any time, without consequences. Participants provided their consent by answering a specific question on their willingness to participate in the study and were subsequently asked to complete the survey. Access to the survey was secure, and the survey software prevented the same user from completing the survey more than once and ensured the anonymity of the participants. Data collection occurred between April, 2014 and June, 2014.

Measures

An online self-report survey was developed for this study, based on a literature review and similar surveys (e.g., Buist et al. 2005; Henshaw et al. 2013; Whitton et al. 1996). The survey was pilot tested with a convenience sample ( $N=10$ women during pregnancy or after childbirth) and items were revised for clarity and comprehensibility.

Participants were asked demographic (age, marital status, educational level, professional status, place of residence and family household income) and clinical information, namely: a) if they were currently pregnant or had given birth during the last 12 months; b) pregnancy information: parity (primiparity vs. multiparity), method of conception (spontaneous vs. medically induced pregnancy), baby's gender, and occurrence of medical complications during pregnancy (yes/no); and c) women's medical history: history of chronic health problems (i.e., persistent physical health conditions, such as asthma, diabetes, cancer; yes/no), history of psychiatric/psychological problems (yes/no) and history of psychiatric/psychological treatment.

To assess women's emotional adjustment, the Portuguese version of the Edinburgh Postnatal Depression Scale (EPDS; Cox et al. 1987; Portuguese version: Areias et al. 1996; Augusto et al. 1996) was used. The EPDS is a widely used 10-item screening scale for antepartum and postpartum depression. Women were asked to rate their emotions (e.g., sadness, tearfulness, anxiety) over the previous seven days, using a 4-point Likert scale. In the Portuguese validation studies, a score of 9 or higher indicates a possible depressive disorder (Areias et al. 1996). In our sample, Cronbach's alpha was .88.

To assess women's help-seeking behaviors, questions were developed based on the existing literature (Cauce et al. 2002; Kadushin 1958). Specifically, participants were asked about different helpseeking behaviors during the perinatal period ("Since you knew that you were pregnant/Since the baby was born...”): a) recognition/awareness of an emotional/psychological problem (“... have you been feeling 
more sad, anxious or stressed out than usual?”; “...do you think that you may be suffering from some psychological problem (e.g., depression, anxiety)?”); b) involvement of the social network (“...have you spoken about the possibility of suffering from a psychological problem (e.g., depression, anxiety) with a family member and/or a friend?"); c) willingness to seek professional assistance for mental health problems (“...have you considered seeking professional assistance in order to handle psychological problems (e.g., depression, anxiety)?"); and d) seeking professional assistance for mental health problems (“...have you sought professional assistance for psychological problems (e.g., depression, anxiety)?”). All questions were answered on a dichotomous response scale (yes vs. no).

To evaluate perceived barriers, participants answered a set of items about attitudinal, knowledge and practical/structural barriers, based on the Barriers Scale (O'Mahen and Flynn 2008) and on a literature review. Women were questioned about the extent to which each item (barrier) would prevent them from seeking professional assistance for their emotional/psychological problems during the perinatal period. The 11 items included knowledge (e.g., "I do not know whether my problems are a reason to seek help"), attitudinal (e.g., "I am afraid of what my family and/or friends might think of me for attending psychology and/or psychiatry appointments"), and practical/structural ("I am not able to afford psychological/psychiatric treatments") barriers. Exploratory factor analyses support the three-dimensional structure of this scale within our sample. An additional item was included regarding perceived barriers not mentioned in the previous items. Items were assessed using a 3-point scale $(0=$ Does not apply to me; $1=$ Moderately applies to me $; 2=$ Very much applies to me .

Data Analyses

Statistical analyses were performed using the Statistical Package for the Social Sciences (IBM SPSS,v.19). Descriptive statistics were used to explore the sample's sociodemographic and clinical characteristics, and to characterize women's help-seeking behaviors and pathways of help-seeking behaviors. Chi-square tests and phi coefficients were used to explore the association between women's help-seeking behaviors.

The association between the sociodemographic and clinical variables and help-seeking behaviors was explored using univariable binary logistic regressions. To determine the sociodemographic and clinical profile of women who had not engaged in any type of help-seeking behavior, a multivariable 
binary logistic regression model was run. The sociodemographic and clinical variables associated with help-seeking behaviors were introduced in the regression model.

Descriptive statistics were also used to assess the number of barriers reported by women and the relative importance of several barriers preventing women from seeking professional assistance. To examine the number of barriers reported by women as a function of type of barriers (attitudinal, knowledge or practical/structural barriers), Friedman tests with Bonferroni corrections $(p<.02)$ were computed. The association between sociodemographic and clinical variables and barriers to seeking professional help was explored using Spearman's rank correlations.

\section{Results}

Participants

A total of 656 women answered the survey, of which $30.2 \%(n=198)$ presented scores above the cutoff on the Portuguese version of the EPDS. Sociodemographic and clinical characteristics of the sample, comparing women who screened positive with those who screened negative for depressive disorders, are presented on Table 1. Most women were married/living together, were currently employed and had given birth during the last 12 months. Compared to women screening negative, those screening positive for depression were younger, were more frequently single/divorced, had a lower monthly household income, and had prior psychiatric history with significantly more frequency.

[Insert_Table_1_about_here]

Help-seeking behaviors

Figure 1 presents the different stages and pathways of help-seeking behaviors of women screening positive for possible depressive disorders during the perinatal period $(n=198)$. Regarding stages, we found that the presence of an emotional/psychological problem was recognized by $40.9 \%$ of women, although a higher proportion $(n=152,76.8 \%)$ of women felt sadder, more anxious or stressed out than usual during the perinatal period (see Figure 1). Moreover, 35.9\% $(n=71)$ of the women discussed the possibility of having a psychological problem with their social network, even when they had not recognized the presence of an emotional/psychological problem. Furthermore, 38.4\% $(n=76)$ of women were willing to seek professional assistance for their emotional/psychological problems, even 
when half of them $(51.3 \%)$ had not recognized the presence of an emotional problem and/or had not discussed it with their social network (see Figure 1). Finally, although 198 women screened positive for possible depressive disorders, only $13.6 \%(n=27)$ of them had sought help for their perinatal depressive symptoms. Even when considering a conservative EPDS cutoff (EPDS $\geq 12$ ), only 20.2\% $(n=22)$ sought professional help for their perinatal depressive symptoms.

[Insert_Figure_1_about_here]

As shown in Figure 1, women presented different pathways of help-seeking behaviors regarding their perinatal depressive symptoms. A significant proportion of women $(n=87,44.0 \%)$ had not engaged in any type of help-seeking behavior (P1), and 9.6\% of women $(n=19)$ presented a pathway characterized by engaging in different help-seeking behaviors (P12). Moreover, women who sought professional assistance reported prior help-seeking behaviors significantly more often: they more often recognized the presence of an emotional problem $\left(\chi_{(1)}^{2}=17.68, p<.001, \Phi=.298\right)$, involved their social network $\left(\chi_{(1)}^{2}=28.29, p<.001, \Phi=.378\right)$, and were willing to seek professional assistance $\left(\chi_{(1)}^{2}=50.19\right.$, $p<.001, \Phi=.503)$

Sociodemographic and clinical predictors of help-seeking behaviors

Table 2 presents the associations between women's socio-demographic and clinical variables and their help-seeking behaviors $(n=198)$.

[Insert_Table_2_about_here]

As shown in Table 2, women who more frequently recognized the presence of an emotional/psychological problem were less educated, were single/divorced, and had history of chronic health conditions and psychological/psychiatric problems. Moreover, women tended to discuss their emotional/psychological problems with a family member and/or a friend and were more willing to seek professional help when they were in the postpartum period and when they had history of psychological/psychiatric problems. Women sought professional assistance more frequently if older, single/divorced, and with a history of psychiatric/psychological problems and treatment (see Table 2). 
The logistic regression model predicting the pathways for women who had not engaged in any type of help-seeking behavior (P1, see Figure 1) was significant $\left(\chi_{(7)}^{2}=16.27, p=.023\right)$ and is presented in Table 3. According to the model, women married/living together, currently pregnant, with no history of psychiatric/psychological problems and a history of psychiatric/psychological treatments had a significantly higher likelihood of not engaging in any type of help-seeking behavior.

[Insert_Table_3_about_here]

Barriers to seeking professional assistance

Among women who had not sought professional assistance $(n=171)$, only $11(5.8 \%)$ did not identify any barrier, and $52.9 \%(n=108)$ of women identified at least five barriers that they considered to be important in preventing them from seeking professional assistance $(M=5.02, S D=2.90)$. Four women reported "other" barriers preventing them from seeking professional assistance (e.g., "My partner does not want me to seek professional help"; "I don't want to take medication"; "No one will help me"). Table 4 presents information about the perceived relative importance of the several barriers. The most frequently identified barriers were related to poor knowledge (about the nature of the mental health problems and treatment options) and practical constraints (financial difficulties and lack of time for psychology/psychiatry appointments). Moreover, women identified significantly more knowledge than attitudinal barriers $(Z=-3.03, p=.002)$, with no differences being found between knowledge and practical barriers $(Z=-1.49, p=.135)$ and between attitudinal and practical barriers $(Z=-1.51, p=.133)$.

[Insert_Table_4_about_here]

Sociodemographic and clinical correlates of perceived barriers to seeking professional assistance

Table 5 presents the sociodemographic and clinical correlates of the perceived importance of the barriers preventing women from seeking professional assistance $(n=171)$.

[Insert_Table_5_about_here]

Globally, attitudinal barriers were perceived as being more important by younger, less educated and unemployed women. Knowledge barriers were perceived as being more important by younger women and by women with no history of psychiatric/psychological problems and treatment; unemployed women believed that not knowing where to seek treatment was an important barrier preventing them from 
seeking professional help. Finally, practical/structural barriers (financial and mobility constraints) were perceived as being more important by younger and less educated women, and by women who were unemployed and who had a lower household income. Women who had medical complications during pregnancy also identified some practical/structural barriers as being important in preventing them from seeking professional assistance.

\section{Discussion}

The present study aimed to contribute to the understanding of women's help-seeking behaviors concerning their perinatal depressive symptoms during the perinatal period. The main findings of this study include the following: a) a significant proportion of women had not engaged in any type of helpseeking behavior regarding their perinatal depressive symptoms; b) women who had not sought professional assistance for their perinatal depressive symptoms perceived knowledge and practical/structural constraints as being important barriers preventing them from seeking help; and c) women currently pregnant, married/living with a partner, with no history of psychological/psychiatric problems and a history of psychiatric/psychological treatments had higher risk of not engaging in any type of help-seeking behavior.

Help-seeking behaviors and barriers to seeking professional assistance

Consistent with prior studies (Dennis and Chung-Lee 2006; Henshaw 2004; McGarry et al. 2009; Patel and Wisner 2011), our results showed that less than 15\% of women screening positive for possible depressive disorders sought professional assistance during the perinatal period. However, similar to other studies (Henshaw et al. 2013; Whitton et al. 1996), a higher proportion of women (approximately $40 \%$ ) engaged in other types of help-seeking behaviors, namely recognizing the existence of an emotional/psychological problem, discussing their emotional/psychological problems with a family member and/or a friend, and being willing to seek professional assistance. This proportion is lower than what is found in other studies (e.g., Henshaw et al. 2013), highlighting the importance of developing efforts to promote different help-seeking behaviors among women during the perinatal period.

To our knowledge, our study was the first to analyze the different paths of help-seeking behaviors in women during the perinatal period, and two main ideas emerge from these results. First, a significant proportion of women screening positive for possible depressive disorders had not engaged in 
any type of help-seeking behavior. These women and their babies have an increased risk of suffering from the pervasive and negative effects of perinatal depression (Field et al. 2010; Kingston et al. 2012; Muzik and Borovska, 2010), to the extent that, despite presenting symptoms of depression, they are not aware of the possibility that they have emotional/psychological problems, and they have not sought informal (family members/friends) or formal (professional) help. Early identification of these women by health professionals is highly important, so that they can be targeted for health interventions that increase their help-seeking behaviors and use of treatment services that are available (McGarry et al. 2009), as discussed below. Second, we found that most women seeking professional assistance for their emotional/psychological problems had engaged more frequently in prior help-seeking behaviors (being aware of the emotional/psychological problems, involving the social network and being willing to seek professional assistance). Therefore, our findings support the idea that the help-seeking process consists of different stages, where individuals tend to gradually move from the personal domain to the interpersonal domain (Rickwood et al. 2005). Furthermore, these results highlight the importance of promoting women's different help-seeking behaviors during the perinatal period, because engaging in other types of help-seeking behaviors increases the women's likelihood of seeking professional assistance for their perinatal depression.

Our results also showed that most women not seeking professional assistance for their emotional/psychological problems report that several barriers were important in preventing them from doing that, which is consistent with prior studies (Bilszta et al. 2010; Callister et al. 2011; Dennis and Chung-Lee 2006; O'Mahen and Flynn 2008). Moreover, our results provide innovative findings about the relative weight of the different barriers preventing women from seeking professional assistance. First, our results showed that knowledge barriers are more frequently identified by women than attitudinal and practical/structural barriers, contrary to O'Mahen and Flynn (2008), who found that women reported being more concerned with practical/structural barriers than with knowledge and attitudinal barriers. Besides reflecting cultural differences, these results suggest the important role that mental health literacy (Jorm 2000) may have in women's help-seeking behaviors during the perinatal period. In addition to knowledge barriers, practical/structural barriers related to financial and time constraints are also perceived by women as being important obstacles; therefore, specific strategies should be developed in order to overcome these barriers. 
Who is at risk of not seeking help?: Sociodemographic and clinical characteristics

Concerning the role of sociodemographic characteristics, our results showed that younger women tend to seek professional assistance less frequently, which is consistent with prior studies (Herrick 2002). In fact, younger women also identified attitudinal, knowledge and practical barriers as being more important in preventing them from seeking professional help, which may explain their less frequent helpseeking behaviors. Moreover, more educated women were found to have more difficulties in recognizing the presence of emotional/psychological problems. Although lower education has been associated with poor mental health literacy in the general population (Kaneko and Motohashi 2007), it is possible that more educated women attribute their symptoms to other causes (e.g., stress related to professional demands) and feel that they are more able to handle the difficulties on their own (Woolhouse et al. 2009), which may impair their ability to recognize emotional/psychological problems. Consistent with these findings, Whitton et al. (1996) found that women of higher social classes are less likely to recognize their symptoms. This hypothesis should be further investigated.

Furthermore, our results also showed that being unemployed, less educated and having a lower household income are associated with the perception that practical and attitudinal issues are important barriers preventing women from seeking professional help, although this does not directly influence helpseeking behaviors. These results reinforce the idea that a disadvantageous socio-economical context is a risk factor for women with perinatal depression, because they may be exposed to several barriers preventing them from seeking professional help.

Finally, women tend to recognize their symptoms and seek professional assistance less frequently when they are married/living with a partner. Single/divorced women may have to deal with the additional demands of being a single parent and, thus, may pay more attention to the emergence of depressive symptoms during the perinatal period and be more willing to ask for help, if necessary.

Some clinical variables were also found to play an important role in predicting women's helpseeking behaviors. First, we found that pregnant women tend to exhibit the different help-seeking behaviors less frequently than women in the postpartum period; however, this variable is not significantly associated with the perceived importance of the several barriers to seeking professional assistance. One possible explanation for these results is that there may be a greater public awareness of depression during the postpartum period than during pregnancy, because postnatal depression is the most widely discussed perinatal mood disorder (McKenzie-McHarg and Cockburn 2007); therefore, women in the postpartum 
period may be more willing to engage in different help-seeking behaviors, whereas pregnant women may hold more unrealistic beliefs about the joyful experience of parenthood.

Second, we found that women who do not have a history of psychiatric/psychological problems and treatment tend to exhibit help-seeking behaviors less frequently, and are also found to identify more knowledge barriers preventing them from seeking professional assistance for their emotional/psychological problems. In fact, women with a psychiatric/psychological history may be more easily able to identify symptoms indicating a possible psychiatric/psychological problem and may be less prone to symptom minimization, denial, and waiting for symptom improvement, because they previously experienced the consequences of having a psychiatric/psychological disorder. These results highlight the important role of education concerning mental health in promoting mental health literacy (Jorm 2000) in the perinatal population.

Finally, our results showed a sociodemographic and clinical profile (being pregnant, being married/living with a partner, having no history of psychiatric/psychological problems and a history of psychiatric/psychological treatment) that significantly predicts the path of women who had not engaged in any type of help-seeking behavior. The early identification of these women may allow for a closer followup of their emotional adjustment over time, as well as the development of strategies specifically targeted to promoting help-seeking behaviors, as discussed below.

Strengths and limitations

This study provided an innovative exploration of women's help-seeking behaviors for perinatal depressive symptoms during the perinatal period by simultaneously considering different types of helpseeking behaviors (paths of help-seeking) and identifying the sociodemographic and clinical characteristics that allow for the early identification of women at higher risk of not seeking help, despite presenting clinically significant depressive symptoms. However, this study has limitations that need to be acknowledged. First, our study was subject to a selection bias, because participation in internet surveys is limited to people who have internet access. It is possible that women without internet access may be even more isolated from support, so this study may not be fully representative of the depression rates and of the help-seeking behaviors of those women. Second, the internet survey was based on a voluntary reply; people willing to participate in this study may be more aware of or concerned with emotional/psychological problems during the perinatal period, so recognition of symptoms and 
willingness for help-seeking may be over-estimated. Clinical settings may be associated with a greater frequency of the help-seeking behaviors that we found in the present study. Third, the cross-sectional nature of the study did not allow for the investigation of patterns of continuity and change in women's help-seeking behaviors during the perinatal period.

Implications for practice

Given the adverse consequences of perinatal depression, mental health professionals have been focusing in the development of effective interventions for this clinical condition. However, as interventions may only be effective if they are used with the target audience, health professionals should focus on minimizing the factors that may prevent women from seeking professional help to deal with their perinatal depressive symptoms during the perinatal period.

Considering this, several implications for practice may be derived from our results. First, the need to improve women's mental health literacy (knowledge about depression symptoms, causes and treatments) during the perinatal period is one important implication of our findings. Education should be provided through community campaigns, as well as by introducing the theme of mental health in childbirth classes and in healthcare appointments during pregnancy and the childbearing years (Callister et al. 2011; Mnich et al. 2014). Second, screening procedures for perinatal depression and an appropriate referral for mental health services should be promoted in healthcare settings, especially among women who present a lower probability of engaging in help-seeking behaviors and should be identified earlier. Third, women should be offered innovative opportunities to network and connect with people and services, by using web-based tools (e.g., institutional websites, web-based support forums; Evans et al. 2012) that promote mental health literacy and encourage the discussion of mental health topics and helpseeking, along with web-based psychological intervention programs (Danaher et al. 2012; Haga et al. 2013) that contribute to overcome the practical barriers (time and financial constraints) that are identified by women as preventing them from seeking professional help. These programs should provide tailored resources that are easily accessible in flexible schedules without additional fees (Fotheringham et al. 2000).

\section{References}

Accortt E, Cheadle A, Schetter C (2015) Prenatal depression and adverse birth outcomes: An updated systematic review. Matern Child Health J 19:1306-1337. doi:10.1007/s10995-014-1637-2 
American Psychological Association (2010) Publication Manual of the American Psychological Association, sixth ed. American Psychological Association, London.

Areias M, Kumar R, Barros H, Figueiredo E (1996) Comparative incidence of depression in women and men, during pregnancy and after childbirth: Validation of the Edinburgh Postnatal Depression Scale in Portuguese Mothers. Brit J Psychiat 169:30-35. doi:10.1192/bjp.169.1.30

Augusto A, Kumar R, Calheiros J, Matos E, Figueiredo E (1996) Post-natal depression in an urban area of Portugal: Comparison of childbearing women and matched controls. Psychol Med 26:135-141. doi:10.1017/S0033291700033778

Beeghly M, Weinberg M, Olson K, Kernan H, Riley J, Tronick E (2002) Stability and change in level of maternal depressive symptomatology during the first postpartum year. J Affect Disorders 71: 169-180. doi:10.1016/S0165-0327(01)00409-8

Bilszta J, Ericksen J, Buist A, Milgrom J, (2010) Women's experience of postnatal depression: Beliefs and attitudes as barriers to care. Aust J Adv Nurs 27:44-54.

Brown S, Lumley J (2000) Physical health problems after childbirth and maternal depression at six to seven months postpartum. Int J Gynaecol Obstet 107: 1194-1201.

Buist A, Bilszta J, Barnett B, Milgrom J, Ericksen J, Condon J, Hayes B, Brooks J (2005) Recognition and management of perinatal depression in general practice: A survey of GPs and postnatal women. Aust Fam Physician 34:787-790.

Byatt N, Biebel K, Lundquist RS, Simas TA, Debordes-Jackson G, Allison J, Ziedonis D (2012) Patient, provider, and system-level barriers and facilitators to addressing perinatal depression. J Reprod Infant Psych 30:436-449. doi:10.1080/02646838.2012.743000

Callister LC, Beckstrand RL, Corbett C (2011) Postpartum depression and help-seeking behaviors in immigrant Hispanic women. JOGNN- J Obstet Gyn Ne 40:440-449. doi:10.1111/j.15526909.2011.01254.x

Cauce AM, Domenech-Rodríguez M, Paradise M, Cochran B, Shea J, Srebnik D, Baydar N (2002) Cultural and contextual influences in mental health help seeking: A focus on ethnic minority youth. J Consult Clin Psych 70:44-55. doi:10.1037/0022-006X.70.1.44

Cox J, Holden JM, Sagovsky R (1987) Detection of postnatal depression: Development of the 10item Edinburgh Postnatal Depression Scale. Brit J Psychiat 150: 782-786. Doi:10.1192/bjp.150.6.782 
Danaher BG, Milgrom J, Seeley JR, Stuart S, Schembri C, Tyler MS, Ericksen J, Lester W, Gemmil AW, Lewinsohn P (2012) Web-Based Intervention for Postpartum Depression: Formative Research and Design of the MomMoodBooster Program. J Med Internet Res. doi: 10.2196/resprot.2329

Dennis CL, Chung-Lee L (2006) Postpartum Depression help-seeking barriers and maternal treatment preferences: A qualitative systematic review. Birth. 33:323-331. doi:10.1111/j.152353X.2006.00130.x

Evans M, Donelle L, Hume-Loveland L (2012) Social support and online postpartum depression discussion groups: a content analysis. Patient Educ Couns. 87:405-410. doi:10.1016/j.pec.2011.09.011

Field T (2010) Postpartum depression effects on early interactions, parenting, and safety practices: A review. Infant Behav Dev 33:1-6. doi:10.1016/j.infbeh.2009.10.005

Field T, Diego M, Hernandez-Reif M (2006) Prenatal depression effects on the fetus and newborn: a review. Infant Behav Dev 29:445-455. doi:10.1016/j.infbeh.2006.03.003

Field T, Diego M, Hernandez-Reif, M (2010) Prenatal depression effects and interventions: a review. Infant Behav. Dev. 33:409-418. doi:10.1016/j.infbeh.2010.04.005

Fotheringham M, Owies D, Leslie E, Owen N (2000) Interactive health communication in preventive medicine: Internet-based strategies in teaching and research. Am J Prev Med. 19:113-120. doi:10.1016/S0749-3797(00)00188-4

Gaynes B, Gavin N, Meltzer-Brody S, Lohr K, Swinson T, Gartlehner G, Brody S, Miller W (2005) Perinatal depression: Prevalence, screening accuracy and screening outcomes. Evidence Report/Technology Assessment. Agency for Healthcare Research and Quality, RockVille.

Goodman J H (2008) Influences of maternal postpartum depression on fathers and on father-infant interaction. Infant Ment Health J. 29:624-643. doi:10.1002/imhj.20199

Goodman JH, Tyer-Viola L (2010) Detection, treatment, and referral of perinatal depression and anxiety by obstetrical providers. J Womens Health. 19:477-490. doi:10.1089=jwh.2008.1352

Haga S, Drozd F, Brendryen H, Slinning K (2013) Mamma mia: A feasibility study of a webbased intervention to reduce the risk of postpartum depression and enhance subjective well-being. J Med Internet Res. 2. doi: 10.2196/resprot.2659

Henshaw CA (2004) What do women think about treatments for postnatal depression? Clinical Effectiveness in Nursing. 8:170-175. doi:10.1016/j.cein.2005.03.003 
Henshaw E, Sabourin B, Warning M (2013) Treatment-seeking behaviors and attitudes survey among women at risk for perinatal depression or anxiety. JOGNN- J Obst Gyn Neo. 42:168-177. doi:10.1111/1552-6909.12014

Herrick H (2002) Postpartum depression: Who gets help? Results from the Colorado, New York, and North Carolina PRAMS Surveys 1977-1999. Statistical Brief No. 24, Department of Health and Human Services. http://digital.ncdcr.gov/cdm/ref/collection/p249901coll22/id/413

Jorm AF (2000) Mental health literacy: Public knowledge and beliefs about mental disorders. Brit J Psychiat. 177:396-401. doi:10.1192/bjp.177.5.396

Kadushin C (1958) Individual decisions to undertake psychotherapy. Admin Sci Quart 3:379-411.

Kaneko Y, Motohashi Y (2007) Male gender and low education with poor mental health literacy: A population-based study. J Epidemiol 17:114-119.

Kingston D, Tough S, Whitfield H (2012) Prenatal and postpartum maternal psychological distress and infant development: a systematic review. Child Psychiat Hum D 43:683-714. doi:10.1007/s10578012-0291-4

Kingston D, McDonald S, Austin M, Tough S (2015) Association between prenatal and postnatal psychological distress and toddler cognitive development: A systematic review. Plos one. doi:10.1371/journal.pone.0126929

McCarthy M, McMahon C (2008) Acceptance and experience of treatment for postnatal depression in a community mental health setting. Health Care Women Int. 29:618-637. doi: $10.1080 / 07399330802089172$

McGarry J, Kim H, Sheng X, Egger M, Baksh L (2009) Postpartum depression and help-seeking behavior. J Midwifery Wom Health. 54:50-56. doi:10.1016(j.jmwh.2008.07.003

McIntosh J (1993) Postpartum depression: Women's help-seeking behaviour and perceptions of cause. J Adv Nurs. 18:178-184. doi:10.1046/j.1365-2648.1993.18020178.x

McKenzie-McHarg K, Cockburn J (2007) Antenatal and postnatal depression. In: Cockburn J, Pawson M (eds.) Psychological challenges in obstetrics and gynecology: The clinical management. Springer, London, pp 141-156.

Mnich E, Makowski A, Lambert M, Angermeyer M, Knesebeck O (2014) Beliefs about depression - Do affliction and treatment experience matter? Results of a population survey from Germany. J Affect Disorders. 164:28-32. doi:10.1016/j.jad.2014.04.001 
Muzik M, Borovska S (2010) Perinatal depression: Implications for child mental health. Mental Health in Family Medicine. 7:239-247.

O'Mahen HA, Flynn HA (2008) Preferences and perceived barriers to treatment for depression during the perinatal period. J Womens Health. 17:1301-1309. doi:10.1089/jwh.2007.0631

Patel SR., Wisner KL, 2011. Decision making for depression treatment during pregnancy and the postpartum period. Depress Anxiety. 28:589-595. doi:10.1002/da.20844

Rickwood D, Deane F, Wilson CJ, Ciarrochi J (2005) Young people's help-seeking for mental health problems. Australian e-Journal for the Advancement of Mental Health. 4:1-34.

Righetti-Veltema M, Conne-Perréard E, Bousquet A, Manzano J (2002) Postpartum depression and mother-infant relationship at 3 months old. J Affect Disorders. 70:291-306. doi:10.1016/S01650327(01)00367-6

Robertson E, Grace S, Wallington T, Stewart DE (2004) Antenatal risk factors for postpartum depression: a synthesis of recent literature. Gen Hosp Psychiatry. 26:289-295. doi:10.1016/j.genhosppsych.2004.02.006

Tronick E, Reck C (2009) Infants of depressed mothers. Harv Rev Psychiat 17:147-156. doi:10.1080/10673220902899714

Van den Bergh BR, Mulder EJ, Mennes M, Glover V (2005) Antenatal maternal anxiety and stress and the neurobehavioural development of the fetus and child: links and possible mechanisms. A review. Neurosci Biobehav Rev 29:237-258. doi:10.1016/j.neubiorev.2004.10.007

Whitton A, Warner R, Appleby L (1996) The pathway to care in post-natal depression: Women's attitudes to post-natal depression and its treatment. Brit J Gen Pract. 46:427-428.

Woolhouse H, Brown S, Krastev A, Perlen S, Gunn J (2009) Seeking help for anxiety and depression after childbirth: Results of the Maternal Health Study. Arch. Women Ment. Health. 12:75-83. doi:10.1007/s00737-009-0049-6

World Medical Association (2000) Declaration of Helsinki: Ethical principles for medical research involving human subjects. JAMA-J AM MED ASSOC. 284:3043-3045. 


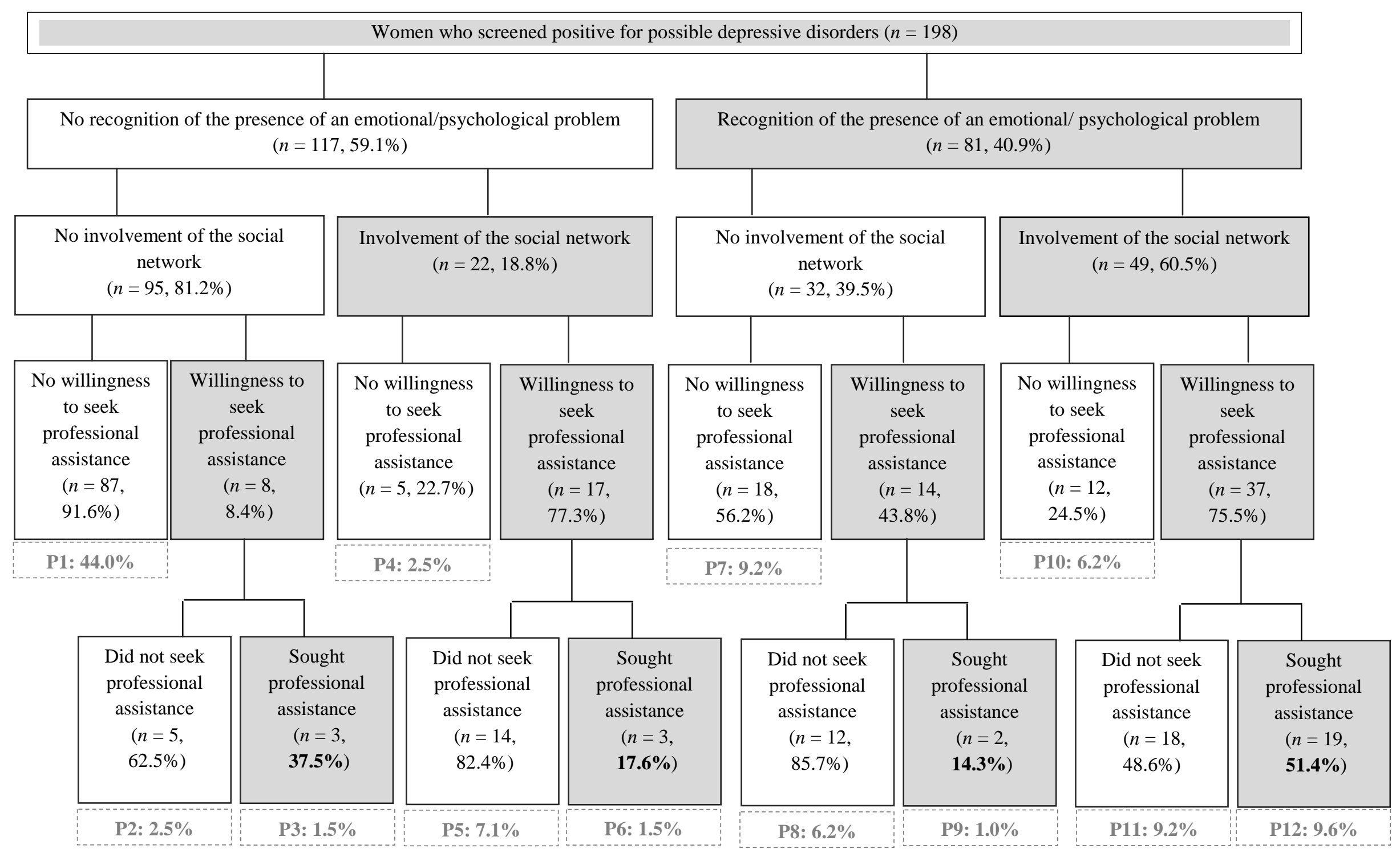

Figure 1. Pathways (P) of help-seeking behaviors of women who screened positive for possible depressive disorders during the perinatal period $(n=198)$ 
Table 1. Sociodemographic and clinical characteristics of the sample

Women $(N=546)$

\section{Sociodemographic characteristics}

Age (in years), $M(S D)$

$31.55(4.09)$

Educational level, $n(\%)$

Middle school

High school

Higher education
$13(2.4)$

$110(22.5)$

$423(77.5)$

Marital status, $n(\%)$

Single $50(9.2)$

Married/living together

$492(90.1)$

Separated/divorced

$4(0.7)$

Professional status, $n(\%)$

$$
\text { Employed }
$$

Unemployed

Other

$17(3.3)$

Monthly Household income, $n(\%)$

$$
\begin{array}{r}
<500 € \\
500-1,000 € \\
1,000-2,000 € \\
2,000-3,500 € \\
>3,500 €
\end{array}
$$

$131(24.0)$

$30(5.5)$

Residence, $n(\%)$
$458(83.9)$

$88(16.1)$

\section{Clinical characteristics}


Pregnancy

Postpartum period

Parity, $n(\%)$
$237(43.4)$

$309(56.6)$

$382(70.0)$

Multiparity

$164(30.0)$

Psychiatric history, $n(\%)$

History of psychiatric/psychological $141(25.8)$

problems (Yes)

History of psychiatric/psychological

$131(25.6)$

treatment (Yes) 
Table 2. Sociodemographic and clinical predictors of women's help-seeking behaviors: univariable logistic regression models $(n=198)$

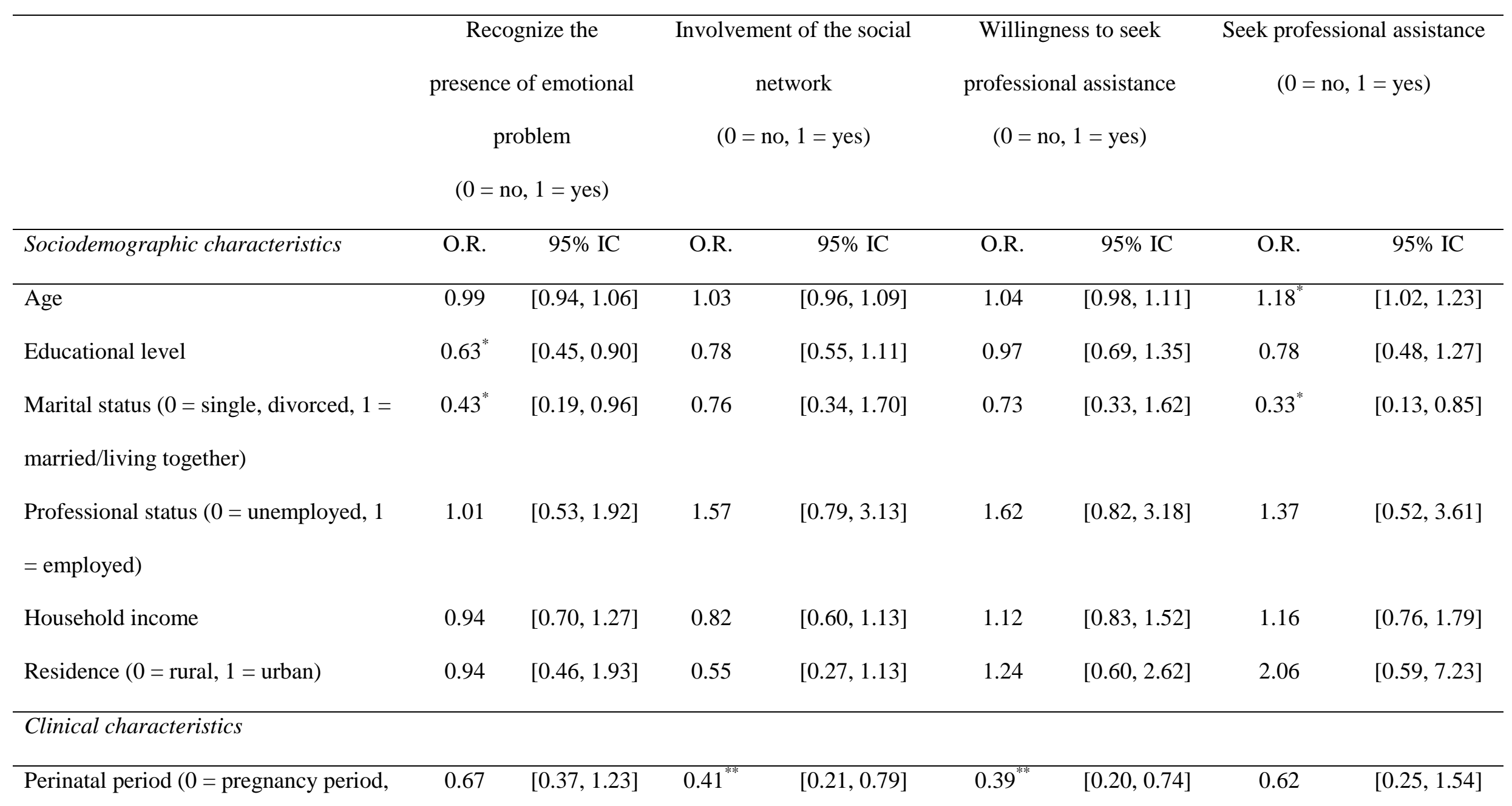




\begin{tabular}{|c|c|c|c|c|c|c|c|c|}
\hline \multicolumn{9}{|l|}{$1=$ postpartum period) } \\
\hline \multicolumn{9}{|l|}{ primiparity) } \\
\hline Medical complications during & 1.43 & {$[0.77,2.69]$} & 1.03 & {$[0.54,1.97]$} & 1.10 & {$[0.58,2.08]$} & 1.36 & {$[0.57,3.24]$} \\
\hline \multicolumn{9}{|l|}{ pregnancy $(0=$ no, $1=$ yes $)$} \\
\hline \multicolumn{9}{|l|}{ yes) } \\
\hline Psychiatric/psychological history $(0=$ & $1.75^{\dagger}$ & {$[0.98,3.13]$} & $2.32^{* *}$ & {$[1.28,4.22]$} & $2.10^{*}$ & {$[1.16,3.79]$} & $4.88^{* * *}$ & {$[2.01,11.83]$} \\
\hline \multicolumn{9}{|l|}{ no, $1=$ yes $)$} \\
\hline
\end{tabular}

${ }^{\dagger} p<.10 .{ }^{*} p<.05 .{ }^{* *} p<.01 .{ }^{* * *} p<.001$. 
Table 3. Logistic regression model with sociodemographic and clinical variables as predictors of the path of women who did not engage in any type of help-seeking behaviors $(n=198)$

\begin{tabular}{|c|c|c|c|}
\hline & OR & $F_{\text {Wald }}$ & $95 \% \mathrm{CI}$ \\
\hline Age & 0.99 & 0.04 & {$[0.92,1.07]$} \\
\hline Educational level & 1.14 & 0.47 & {$[0.78,1.66]$} \\
\hline $\begin{array}{l}\text { Marital status }(0=\text { single, divorced, } 1= \\
\text { married/living together })\end{array}$ & 2.41 & $3.18^{\dagger}$ & {$[0.92,6.34]$} \\
\hline $\begin{array}{l}\text { Professional status }(0=\text { unemployed, } 1= \\
\text { employed })\end{array}$ & 0.80 & 0.38 & {$[0.39,1.65]$} \\
\hline $\begin{array}{l}\text { Perinatal period }(0=\text { pregnancy period, } 1= \\
\text { postpartum period })\end{array}$ & .52 & $4.05^{*}$ & {$[0.27,0.98]$} \\
\hline Chronic health conditions $(0=$ no, $1=$ yes $)$ & 0.83 & 0.24 & {$[0.39,1.76]$} \\
\hline Psychiatric/psychological history $(0=$ no, 1 & 0.38 & $4.77^{*}$ & {$[0.16,0.91]$} \\
\hline yes) & & & \\
\hline $\begin{array}{l}\text { History of psychiatric/psychological } \\
\text { treatment }(0=\text { no, } 1=\text { yes })\end{array}$ & 2.08 & $2.87^{\dagger}$ & {$[0.89,4.86]$} \\
\hline
\end{tabular}

Note. Dependent variable: 1 = path of women who did not engage in any type of help-seeking behaviors (P1, see Figure 1), $0=$ other paths. -2 Log-Likelihood $=245.87$; Pseudo $R^{2}=.073$ (Cox \& Snell), .098 (Nagelkerke). Hosmer \& Lemeshow goodness-of-fit test $=6.59, p=.582$. 
Table 4. Barriers to seeking professional assistance: women's perception of relative importance $(n=171)$

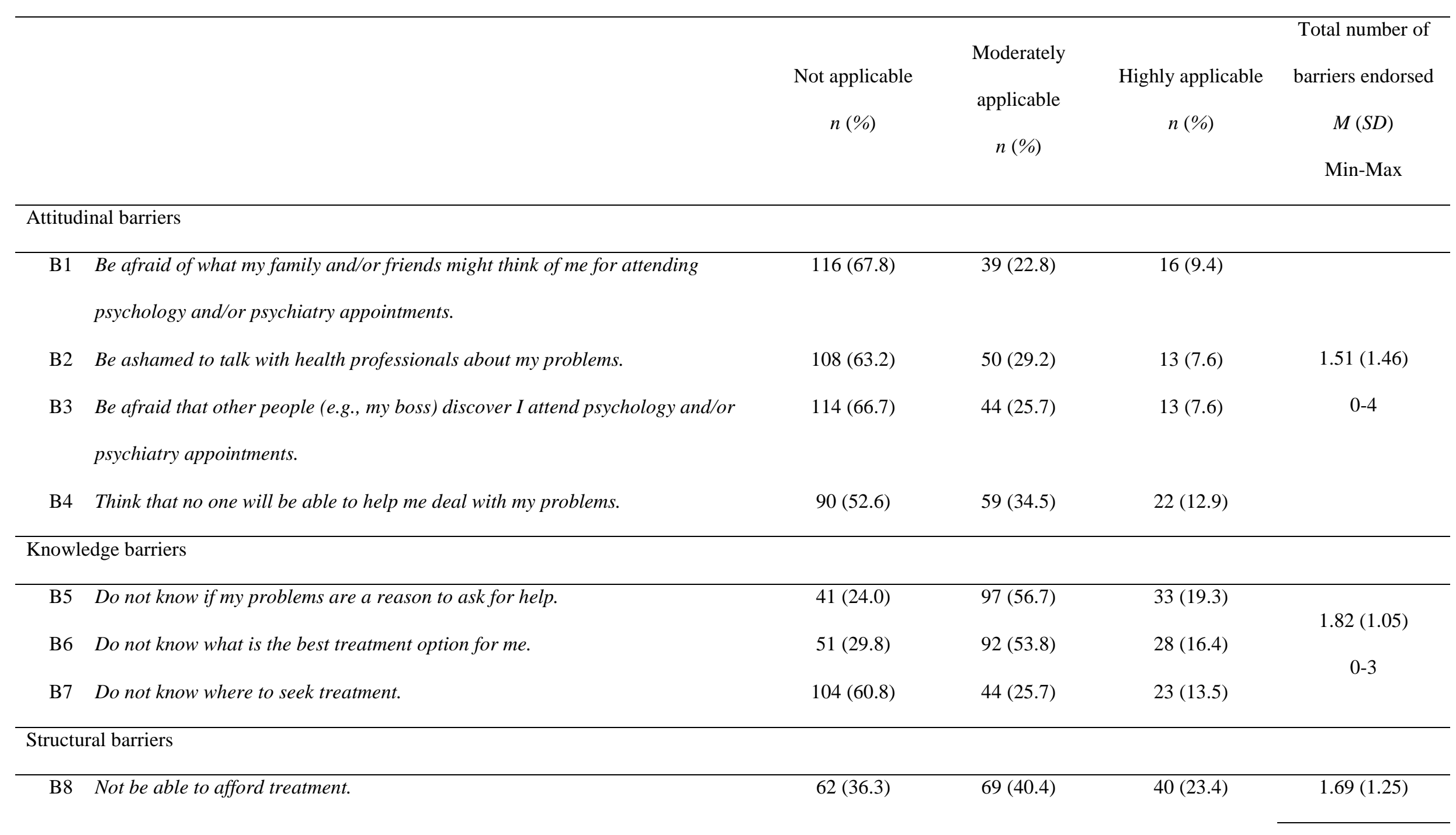


B9 Do not have time to go to psychology and/or psychiatry appointments.

B10 Have sanctions for missing work to go to psychology and/or psychiatry appointments (e.g., earning less money, being fired).

B11 Do not have means to travel to psychology and/or psychiatry appointments.

$84(49.1) \quad 56(32.7) \quad 31(18.1)$

$105(61.4)$

$42(24.6)$

$24(14.0)$

$138(80.7)$

$23(13.5)$

$10(5.8)$ 
Table 5. Sociodemographic and clinical correlates of women's perceived importance of barriers to seek professional assistance $(n=171)$

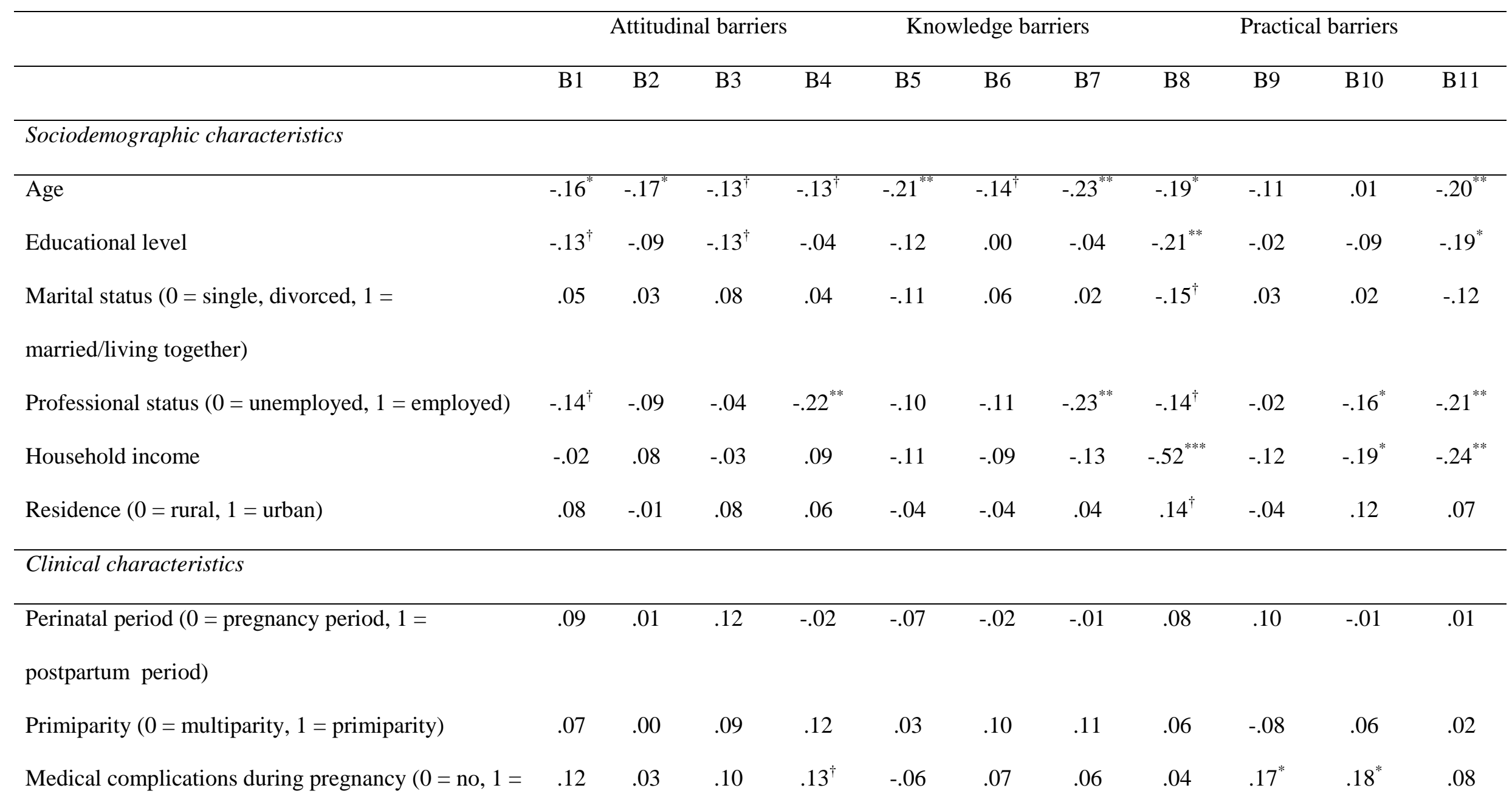


yes)

Chronic health conditions $(0=$ no, $1=$ yes $)$

Psychiatric/psychological history $(0=$ no, 1 yes $)$

$-.06$

History of psychiatric/psychological treatment $(0=$

$-.07 \quad-.09$

$-.01--.08$

$01-.12$

$-12-.18$

$-.08$

$-.14^{\dagger}-.15$

$-09$

$.15^{*}$

.05

.03

no, 1 = yes)

${ }^{\dagger} p<.10 .^{*} p<.05 .^{* *} p<.01 .^{* * *} p<.001$. 